\title{
Multilateral für die EU als Friedensmacht, für Klimaschutz und Demokratie
}

\author{
Omid Nouripour
}

Eingegangen: 1. Februar 2021 / Angenommen: 3. März 2021 / Online publiziert: 19. Mai 2021

(C) Der/die Autor(en) 2021

Zusammenfassung Der Beitrag argumentiert, dass die Herausforderungen dieser Zeit, wie der Klimawandel, nur in multilateraler Zusammenarbeit gemeistert werden können. Die zentralen Orte der Zusammenarbeit bleiben für Deutschland die EU und die UN. Die EU muss ihre strategische Souveränität in den kommenden Jahren stärken und entschiedener den Wettbewerb im Rahmen der systemischen Rivalität mit Akteuren wie China und Russland führen. Geleitet werden sollte sie dabei von der Handlungsmaxime: selbstbewusste Kooperationsbereitschaft, wo immer möglich, und eigenständigem Handeln, wo immer nötig.

Schlüsselwörter Sicherheitspolitik · Multilaterale Zusammenarbeit · Europäische Union · Vereinte Nationen · Klimawandel

\section{A Multilateral Commitment to the EU as a Force for Peace, for Climate Protection and Democracy.}

\begin{abstract}
This article argues that the challenges of this time, such as climate change, can only be overcome through multilateral cooperation. For Germany, the central places of cooperation remain the EU and the UN. The EU must strengthen its strategic sovereignty in the coming years and compete more decisively in the context of systemic rivalry with actors such as China and Russia. In doing so, it should be guided by the principle of self-confident willingness to cooperate wherever possible, and independent action wherever necessary.
\end{abstract}

Keywords Security policy · Multilateral cooperation · European Union · United Nations $\cdot$ Climate change

O. Nouripour, MdB ( $\square)$

Fraktion Bündnis 90/Die Grünen im Deutschen Bundestag, Platz der Republik 1, 11011 Berlin,

Deutschland

E-Mail: omid.nouripour@bundestag.de 


\section{Einleitung}

Vor vier Jahren veröffentlichte die Zeitschrift für Außen- und Sicherheitspolitik (ZfAS) traditionell in ihrer zweiten Ausgabe des Bundestagswahljahres Beträge von führenden Politiker*innen Deutschlands über die Außenpolitik. Für meine Partei Bündnis 90/Die Grünen führte unsere Fraktionsvorsitzende im Deutschen Bundestag Katrin Göring-Eckardt in die Grundzüge der Positionen der Partei in der Außenund Sicherheitspolitik ein. Liest man jetzt, vier Jahre später, ihren Beitrag mit der Überschrift „Multilaterale Kooperation als Antwort auf globale Krisen“, so ist Göring-Eckardts Einwurf weiterhin von großer Richtigkeit und Aktualität.

Die Zusammenfassung dessen in der ZfAS lautete wie folgt:

Der Beitrag stellt die Außen- und Sicherheitspolitik von Bündnis /Die Grünen vor. Es wird argumentiert, dass die Antwort auf globale Herausforderungen nur in verstärkter multilateraler Kooperation liegen kann. Deutschland muss sich für eine starke Europäische Union einsetzen und mehr internationale Verantwortung für Frieden, Menschenrechte und nachhaltige Entwicklung im Rahmen der Vereinten Nationen übernehmen.

Dies hat weiterhin bestand. Wir Grüne setzen auf eine Politik, die sich in Kooperation ihrer globalen Verantwortung stellt. Unsere Definition von Kooperation ist dabei nicht auf Regierungszusammenarbeit beschränkt, sondern auch auf subnationale Ebenen. Die Auswärtige Kultur- und Bildungspolitik ist unsere Brücke in die Zivilgesellschaften der Welt. Dabei sind Menschenrechte unverhandelbar, zentral dabei ist die Feststellung, dass Frauenrechte Menschenrechte sind. Auch die Nachhaltigkeitsziele der Vereinten Nationen mit ihrem bindenden Völkerrechtscharakter sind für uns handlungsleitend.

Die Grünen setzen sich für eine Politik ein, die sich von der Verwirklichung von Frauen- und Minderheitenrechten, wie zum Beispiel die Rechte von inter- und transgeschlechtlichen Menschen, dem Schutz vor geschlechtsspezifischer, rassistischer und anderer menschenfeindlicher Gewalt, Verfolgung und Diskriminierung sowie einer aktiven Unterstützung und die Befähigung zur Selbstbestimmung von Mädchen, Frauen und anderen marginalisierten Gruppen in allen Bereichen leiten lässt. Das geht nur international und beginnt im vereinigten Europa, das als einzigartiges Friedensprojekt als Lehre aus zwei Weltkriegen und der Shoa entstanden ist, und eine Mitverantwortung für Frieden weltweit trägt. Für die Grünen sind internationale Solidarität sowie Verantwortung für unser historisches und heutiges Handeln zentral. Diese sind nur mit einer weltweiten Ordnung mit internationalen Institutionen erreichbar.

Bei genauerer Lektüre des Beitrags Göring-Eckardts stellt man vier Jahre später fest, dass sich die Zeilen aus dem Jahr 2017 wie aus einem anderen Jahrhundert anfühlen. Denn die Schnelllebigkeit weltweiter Ereignisse hat eine Dynamik in die internationale Politik gebracht, wie wir sie noch nie erlebt haben. Und doch bleiben die formulierten Grundsätze richtig. Die Herausforderungen aber ändern sich rapide.

Es lohnt sich also, diese Herausforderungen genauer unter die Lupe zu nehmen. Und die größten für die deutsche Außen- und Sicherheitspolitik lassen sich unter 
zwei Überschriften zusammenfassen: der Klimawandel und die systemische Rivalität.

\section{Klimawandel als Brandbeschleuniger}

Der Klimawandel bedroht nicht erst seit neuestem unsere Gewohnheiten, unseren Wohlstand und unsere Gewissheiten. Er bedroht zunehmend für alle spürbar die Lebensgrundlagen vieler Menschen. Damit verschärft er bereits existierende Verteilungskämpfe massiv und befeuert bereits existierende Konflikte. Dass Bashar alAssad 2011 auf friedliche Demonstrierende im Süden Syriens hat feuern lassen, dass die Proteste der Menschen in Syrien sich in erster Linie gegen Unfreiheit und Korruption richteten und bis heute richten, hat mit dem Klimawandel nichts zu tun. Dass der Leidensdruck, gerade der Landbevölkerung, nach harten Dürrejahren aber das Fass mit zum Überlaufen gebracht hat, dagegen schon.

„Foreign Policy is Climate Policy“ ist der Titel einer hervorragenden gemeinsamen Studie von Adelphi und dem Wilson Center. Außenpolitik und damit verbunden Klimadiplomatie ist in der Tat auch als Instrument für Klimaschutz zu verstehen. Sie ist deshalb eine zentrale Aufgabe für die internationale Politik Deutschlands im 21. Jahrhundert. Denn kein Land der Welt kann allein der Herausforderung des Klimawandels begegnen. Darüber gibt es keine Zweifel mehr. Diese Erkenntnis führt auch dazu, dass es im Klimabereich eine so dringende Notwendigkeit der Kooperation gibt wie kaum zuvor. Nicht umsonst hat die amerikanisch-russische Zusammenarbeit in der Arktis in den letzten Jahren nie pausiert - egal wie groß die Spannungen seit dem russischen Feldzug in der Ukraine waren.

Besonders relevant ist das Thema Klimakooperationen vor allem in Bezug auf China. Der EU-Dreisatz über China von Mitbewerber, Partner, systemischer Rivale lässt sich auch zuspitzen auf Markt-Wettbewerber, Klimapartner, dauerhafter systemischer Rivale.

\section{Bedrohliche systemische Rivalität}

Damit sei die zweite große Herausforderung unserer Zeit genannt: Wir sind nicht in einem neuen Kalten Krieg. China ist nicht die Sowjetunion - Russland schon gar nicht. Aber spätestens seit dem Beginn der Pandemie hat die Führung der Volksrepublik den Wettbewerb der Systeme laut und offiziell ausgerufen. Dabei ist die Kommunistische Partei sehr darauf bedacht, nach innen wie nach außen die Überlegenheit und die Effizienz ihres eigenen Herrschaftssystems unseren Demokratien gegenüber zu unterstreichen. Längst sprechen die Funktionäre aus Peking bei jeder Gelegenheit über das Post-West-Zeitalter und weisen auf ein angebliches Scheitern unserer Demokratien hin. Diesen Wettbewerb ernst und selbstbewusst anzunehmen, das ist eine der zentralen Aufgaben deutscher (Innen)- und Außenpolitik unserer Zeit.

Dabei kommt dem Zusammenhalt und der Wirkungsmächtigkeit der Europäischen Union eine zentrale Rolle zu; aber auch einem Neustart der transatlantischen 
Beziehungen. Das Verhältnis zwischen uns und den Amerikanern war nie spannungsfrei, doch stets wertebasiert und von gegenseitigem Respekt geprägt. Genau dies aber fehlte unter dem ehemaligen US-Präsident Donald Trump. Und es ist denkbar, dass ähnliche Zeiten wieder einkehren können. Auch deswegen muss Europa auf eigenen Beinen stehen und seine Souveränität verteidigen können. Dennoch ist gerade die systemische Rivalität gemeinsam mit den anderen großen Demokratien der USA und Kanadas, aber auch mit Partnern in Asien, Ozeanien, Afrika und Lateinamerika bei weitem leichter zu bestehen.

Die Erneuerung der Wertepartnerschaft zwischen der EU und den USA nach der Wahl von US-Präsident Joe Biden und US-Vizepräsidentin Kamala Harris steht vor der großen Aufgabe der Pandemiebekämpfung, aber auch der ökologischen Transformation unserer Gesellschaften. Ein verbindendes Element bei der Bewältigung dieser Aufgabe ist unser gemeinsamer Einsatz für die Stabilisierung und Stärkung unserer Demokratien. Der Europäische Green Deal passt dabei sehr gut zur neuen Prioritätensetzung der USA, Klimaschutz mit Kraft voranzutreiben und mit sozialem Ausgleich und ökonomischer Prosperität zu verbinden.

Der Anspruch, mit dem notwendigen Strukturwandel stärker aus der Pandemie herauszukommen, ist eine gute Arbeitsgrundlage für die Kooperation in den internationalen Beziehungen. Das kann und muss allerdings über die transatlantischen Beziehungen hinaus erfolgen. In den verlorenen vier Jahren der Trump-Präsidentschaft hat sich gezeigt, dass Europa eine geopolitische Rolle hat, die kaum ausgefüllt wurde. Das daraus entstandene Vakuum haben dann andere geschlossen - auf Kosten der Europäer, auch in unserer unmittelbaren Nachbarschaft.

\section{Die bisherige Abwesenheit der Europäer}

Heute entscheiden Russland, Iran und die Türkei den Kurs in Syrien. Sicher nicht einvernehmlich, aber doch in Abwesenheit der Europäer. In Libyen sind es Russland, die Türkei und die Vereinigten Arabischen Emirate, deren Wort zählt. Deutschlands einzige Initiative hierbei war die Berliner Konferenz, deren Ergebnisse von den genannten Staaten schlicht ignoriert werden, ohne dass es für sie folgen hätte. In BergKarabach haben die Türkei und Russland den Krieg im Jahr 2020 entschieden. Und dass die Europäer nach dem Ausstieg der Trump-Administration aus dem Atomdeal mit dem Iran nicht den Mut hatten, sich der US-Politik beherzt entgegenzustellen, obwohl es um die Verhinderung der Nuklearisierung unserer Nachbarschaft im Nahen Osten geht, stellt dem europäischen Streben nach einer eigenständigen Außenund Sicherheitspolitik ein noch schlechteres Zeugnis aus. Viele weitere Beispiele könnten genannt werden. Die Mutlosigkeit in der Westbalkanpolitik mit der daraus resultierenden Zunahme chinesischer Einflussnahme, oder aber auch die Uneinigkeit der EU in der Frage, wieviel Druck auf den belarussischen Diktator Alexander Lukaschenko notwendig ist, um ihn von der harten Repressionswelle gegen die eigene Bevölkerung abzuhalten.

Das Jammern über Europas Handlungsfähigkeit ist nichts Neues. Und es bringt uns nicht weiter. Ja, das Prinzip der Einstimmigkeit muss schrittweise durch Mehrheitsentscheidungen ersetzt werden, um die EU handlungsfähiger zu machen. Selbst- 
verständlich ist mehr in der Östlichen Partnerschaft der EU möglich wie auch in der Südlichen. An dieser Stelle sei aber auch erwähnt, dass die EU in der Außenpolitik durchaus einiges erreicht hat in den letzten Jahren. Auch deshalb, weil die gemeinsamen europäischen Institutionen wie die Organisation für Sicherheit und Zusammenarbeit in Europa (OSZE) oder der Europarat im Zusammenspiel mit der EU wichtige Plattformen einer multilateralen Weltordnung sind.

Aber entscheidend ist, dass wir besser werden müssen, um weltpolitikfähig werden zu können. Und dass das Ende von Trump uns eine zweite Chance gibt. Die Prämisse für diese Chance auf eine neue transatlantische Zusammenarbeit ist eine EU, die handlungsfähig ist.

\section{Europa kann mehr}

Denn Europa kann mehr, als es bisher zeigt: Der europäische Binnenmarkt ist der größte Wirtschaftsraum der Welt, dessen Wirtschaftskraft gewaltig ist. Wir haben eine größere Wirtschaftsleistung als die USA oder China. Kein großes Unternehmen kann es sich erlauben, auf unserem Markt nicht vertreten zu sein. Der EU kommt eine Schlüsselrolle im globalen Machtgefüge zu. Aber der Riese EU verhält sich oft wie ein Zwerg. Es gibt keinen Grund, gegenüber China vor Angst zu erstarren oder vor den Trumps dieser Welt zu kapitulieren. Die Erzählung, dass die EU zwischen China und den USA zerrieben wird, ist kein Naturgesetz, das man einfach hinnehmen muss. Das geht vor allem, wenn die EU ihre wirtschaftliche Stärke ebenfalls als geostrategisches Instrument begreift. Nicht als Mittel der Erpressung postkolonialer Akteure, sondern als Zeichen der eigenen Befähigung und des eigenen Selbstbewusstseins. Europa kann eigene Regeln und Standards setzen und so souverän und weltpolitisch handlungsfähig sein.

Diese Handlungsfähigkeit ist notwendig für den Erhalt, ja teilweise zur Wiederherstellung der Souveränität Europas. Wenn die Türkei und die Vereinigten Arabischen Emirate ihren weltanschaulichen Konflikt in europäische Gewässer im östlichen Mittelmeer tragen, wenn strategisch relevante Häfen in Europa mehrheitlich in chinesischer Hand liegen, wenn die Lufthoheit über dem Schwarzen Meer von Russland diktiert wird, wenn das US-Finanzministerium bestimmt, mit wem europäische Firmen Geschäfte machen dürfen, dann ist die Souveränität Europas mehr als gefährdet. Diese wieder zu erlangen, ist zentral. Nicht im Sinne von Abschottung, schon gar nicht im Sinne von Autarkie oder einer angeblichen Autonomie, die in einer globalisierten Welt niemand hat und sich auch kein Land leisten kann. Es geht um eine Europäische Union, die als Friedensmacht kooperativ und handlungsfähig ist. Es geht bei der strategischen Souveränität Europas also um selbstbewusste Kooperationsbereitschaft, wo immer möglich und eigenständigem Handeln, wo immer nötig. Denn gut vernetzt zu sein, ist auch für Staaten Notwendigkeit, um handlungsfähig zu sein im 21. Jahrhundert.

Die EU hat diese Aufgabe in der Tat nun aufgenommen und einiges in Gang gesetzt. Es braucht aber nicht nur eine Kommission, die beispielsweise eine Konnektivitätsstrategie als Gegenentwurf zur chinesischen Seidenstraßeninitiative vorantreibt, sondern auch EU-Mitgliedsstaaten, die Brüssel dabei beistehen. Die Seidenstraße 
sei hier als anschauliches Beispiel dafür genannt, wie Peking durch infrastrukturelle Vernetzung strategische Abhängigkeiten schafft, die sie ökonomisch wie politisch jederzeit ausschöpfen kann. Dass die Kritik Londons am völkerrechtswidrigen Wirken Chinas in Hongkong zum Stopp der Bauarbeiten von Kraftwerken in Großbritannien führte, ist nur ein Beispiel hierfür. Die Initiative der EU allerdings basiert auf einem Wertesystem frei von Willkür und Erpressung.

Doch es braucht mehr als nur diese Initiative. Der Green Deal der EU zeigt in die richtige Richtung. Er kann eine klimaneutrale Wirtschaft auf den Weg bringen, unsere Industrie fit für die Zukunft machen und zur globalen Gerechtigkeit beitragen - je nachdem, wie er genau konfiguriert wird. Und er kann an dem doch sehr ambitionierten Programm der Biden/Harris-Aministration anknüpfen, 1,9 Billionen US-\$ in Erneuerbare Energien, Infrastruktur und Mobilität, ins Gesundheitssystem, sozialen Wohnungsbau und in Schulen und Kitas zu investieren.

\section{Die Vereinten Nationen als zentrale Plattform globaler Zusammenarbeit}

Die neue Regierung in Washington gibt uns allerdings auch in einem anderen Bereich eine neue Chance. Viel ist seit Beginn der Pandemie gesagt und geschrieben worden über den (zu) großen Einfluss Chinas auf die Weltgesundheitsorganisation (WHO). Auch hier ist bemerkbar, dass Chinas Einfluss mit dem Rückzug westlicher Demokratien, allen voran der USA aus den supra- und internationalen Organisationen erst ermöglicht wurde. Dass Trump deshalb die USA aus der WHO zurückgezogen hat, ist genau die falsche Konsequenz aus dieser Feststellung. Wenn es stimmt, dass globale Probleme nur mit globaler Zusammenarbeit zu lösen sind, dann müssen die westlichen Staaten, dann müssen die Europäer, dann muss Deutschland sich weit mehr einbringen in die internationalen Organisationen; personell, finanziell und politisch. Noch einmal exemplarisch an der WHO durchbuchstabiert: Die EU muss jetzt, am besten in Kooperation mit den USA, eine Initiative zur Reform und Stärkung der WHO starten. Ziel muss die Erhöhung ihrer Unabhängigkeit und Durchschlagfähigkeit sein. Gelingen wird das aber nur, wenn wir als Europäer, als der Westen, als die reichen Länder, uns weit hilfsbereiter zeigen bei der Bewältigung der Pandemie. Die solidarische Verteilung von medizinischer Ausrüstung und Medikamenten gehört da genauso dazu, wie der bezahlbare Zugang zum Impfstoff für alle. Dabei sollte die Solidarität mit ärmeren Staaten im Fokus stehen. Dies ist nicht nur eine Frage der Moral oder eine der Eindämmung der Pandemie in einer globalisierten Welt. Es ist auch eine Frage knallharter Geostrategie. Denn die Vereinten Nationen bleiben die zentrale Plattform notwendiger globaler Zusammenarbeit auf allen Ebenen.

\section{Anschluss verpasst}

Zurück zum Green Deal. Das wichtigste Ziel der Politik für die nächsten Jahre ist das Erreichen der Klimaneutralität. Ohne die Realisierung dieses Ziels wird Klimaschutz bald kaum mehr möglich sein. Dies muss das klare Signal des kommenden 
Klima-Gipfels der UN in Glasgow im November 2021 sein: ambitionierte CO2-Einsparziele, gepaart mit beständiger Finanzierung der Mittel und klar überprüfbarer Wegmarken dahin. Dafür müssen die EU und die USA zusammenstehen. Gerade in Zeiten der Rückkehr der Kohlekraftwerke in China ist es wichtig, dass der transatlantische Raum mit einem 40\%igen Anteil an der globalen Wirtschaftskraft vorangeht. Das geht über den Ausbau der Wasserstoffinfrastruktur zur Reduzierung von Prozessemissionen für klimaneutralen Stahl und Zement genauso wie gemeinsame Standards, Finanzierungsmodelle und den Ausbau von Produktionskapazitäten für Batterien, Batterierecycling und Ladeinfrastruktur von Elektromobilität. Dabei ist es, wie eingangs beschrieben, notwendig, China als Klimapartner zu gewinnen; auch Drittstaaten gegenüber. Die Finanzierung von Kohlekraftwerken in Schwellenstaaten hilft der Menschheit nicht - egal wer der Financier ist. Der Klimawandel ist das vielleicht beste Beispiel für die anzustrebende Balance zwischen souveräner Handlungsfähigkeit und dem internationalen Kooperationsgebot. Aber es gibt noch andere: Europa hat im letzten Jahrzehnt den digitalen Anschluss an die Weltspitze schlicht verpasst. Investitionen in Energienetze, moderne Hochgeschwindigkeitszüge und die Datenautobahn - all diese Infrastrukturen müssen öffentlich und dringend finanziert werden. Unsere Wettbewerbsfähigkeit wiederherzustellen, ist eine zentrale Aufgabe nicht nur für unseren Wohlstand, sondern auch für die Stellung Europas in der Welt. Aber auch für den Erhalt unserer Datenschutzstandards. Auch deshalb ist die Zusammenarbeit mit den USA in diesem Bereich wohl schwieriger als auf jedem anderen Feld. Gerade bei uns in Deutschland ist Privatsphäre ein Element der Menschenwürde. In den USA allerdings wird Privacy in erster Linie als ein Aspekt des Schutzes des Eigentums verstanden. Und dass die technologische Überwachung à la Kommunistische Partei Chinas nichts mit unserem Verständnis von Privatsphäre und dem damit verbundenen Schutz der Bürger*innen vor dem Staat zu tun hat, versteht sich von selbst. Technologisch jedoch ist Europa so sehr im Verzug, dass auch hier gelten muss: so viel Kooperation wie nötig. Die anzustrebende digitale Souveränität Europas jedoch braucht erhebliche Kraftanstrengungen, finanziell wie intellektuell. Und auch hier gilt, dass die Potenziale einer einheitlichen EU-Linie im Schulterschluss mit den USA gewaltig sind: Sollten sich die EU und USA auf eine Regulierung und Besteuerung der GAFAM (Google, Amazon, Facebook, Apple und Microsoft) einigen, sollten wir zu gemeinsamen Richtlinien kommen bei der Bekämpfung von Hass und Fake News. Eine Zusammenarbeit in diesem Bereich würde globale Maßstäbe setzen, deren Bedeutung nicht zu unterschätzen ist. Das wäre auch ein hoffnungsvolles Fundament für eine gemeinsame Zusammenarbeit etwa bei der Künstlichen Intelligenz (KI) oder gar der Künstlichen Allgemeinen Intelligenz (Artificial General Intelligence, AGI). Es ist allerdings abzuwarten, ob die Biden-Regierung ihre Nähe zu Silicon Valley eher zur klassischen nationalen Industriepolitik entwickelt, was die Zusammenarbeit mit Europa in diesem Bereich eher erschweren dürfte. Die Regulierung des Cyberspace ist eine weitere zentrale Aufgabe, die nur gemeistert werden kann, wenn sie als Teil einer (bisher fehlenden) Global Governance verstanden wird. Die alte deutsch-brasilianische UN-Initiative in diese Richtung war richtig, wurde aber leider nicht beherzt weiterverfolgt. Dies müssen wir ändern. Doch auch hier gilt, dass die EU nur dann global handlungsfähig ist, wenn sie europäisch handlungsfähig ist. Dafür braucht es nicht nur den Willen, son- 
dern auch die richtigen Instrumente und Institutionen. Denkbar wäre beispielsweise ein Europäisches Cybersicherheit-Zentrum, auf der Grundlage des bereits existierenden Kompetenzzentrums für Cybersicherheit. Doch der Schutz von Demokratie und Rechtsstaatlichkeit beschränkt sich bei weitem nicht auf das Internet. Und auch hier gilt es, die Aufgaben im eigenen Haus in den Mittelpunkt zu stellen. Die Erosion demokratischer Checks and Balances in der Europäischen Union zu bekämpfen, ist dabei der erste Schritt. Die Errichtung des Rechtstaatlichkeitsmechanismus ist dafür ein guter Ausgangspunkt. Doch auch hier gilt es, den Schulterschluss der Demokratien weltweit zu suchen. Eben auch wegen der systematischen Rivalität mit den illiberalen und repressiven Autokratien.

Dies gilt auch für den Handel. Natürlich bedarf es weiterhin der wirtschaftlichen Vernetzung mit China. Das Trumpsche Fabulieren von Decoupling war weder realitätstauglich noch friedensstiftend. Doch dabei muss es klare Standards geben, gerade im Menschenrechtsbereich. Ein Negativbeispiel ist sicherlich das kürzlich ausgehandelte EU-China-Investitionsabkommen, das die Tatsache millionenfacher Zwangsarbeit, gerade der uigurischen Minderheit im Land, schlicht ausblendet.

\section{Fairer und freier Handel}

Auch in Handelsfragen mit China braucht es mehr europäischen Zusammenhalt. Dass Handelsfragen in der Kompetenz der EU-Kommission liegen, ist hierbei hilfreich. Doch müssen auch die Mitgliedsstaaten aufmerksamer Direktinvestitionen in Europa, die wirtschaftliche und politische Abhängigkeiten schaffen, verfolgen und besonders in Bereichen der kritischen Infrastruktur unterbinden oder zumindest Reziprozitätsmöglichkeiten schaffen. Hilfreich dabei ist im Übrigen auch ein Mapping gebrochener Investitionsversprechen Chinas. Diese sind zahlreich und zeigen den Unterschied zwischen Schein und Sein doch sehr deutlich. Dabei dürfen wir grundsätzlich nicht vergessen, wie groß Handelsfragen als Hebel der Umsetzung eigener europäischer Standards sein können. Deshalb ist es notwendig, dass die EU keine Freihandelsabkommen mehr abschließt, die nicht die Pariser Klimaziele und die Kernarbeitsnormen der International Labour Organization (ILO) zum zentralen Bestandteil haben. Denn nur ein fairer Handel ist ein freier Handel. Es ist aber auch klar: der zunehmenden Aushöhlung des Multilateralismus - die sich in bilateralen Deals zeigt - müssen wir als EU etwas entgegensetzen und das geht vor allem mit Handelsabkommen. Es ist in unserem Interesse, in einem Netz von Handelsabkommen europäische Standards zu setzen und multilateraler Kooperation wieder eine Chance zu geben. Europa darf nicht das schleifen, was es stark gemacht hat: ein starkes Wettbewerbsrecht. Weitere Schritte müssen folgen, wie etwa eine europäische statt einer nationalen Industriestrategie. Auch große Unternehmen können nicht mit hochsubventionierten chinesischen Staatskonzernen konkurrieren. Deshalb bedarf es einer abgestimmten europäischen Politik, die mit Antidumpingmaßnahmen reagiert - beispielsweise mit einem echten CO2-Preis mit klimaschützender Wirkung. Aber auch andere Erfolgsmodelle haben Maßstäbe gesetzt: Das Verfahren der EU-Kommission gegen Google etwa, das mit einer Rekordkartellstrafe in Milliardenhöhe abgeschlossen wurde. Hätten einzelne EU-Mitgliedsstaaten das versucht, 
wären sie gewiss gescheitert. Auch deshalb sei hier das vielleicht dickste zu bohrende Brett auf dem Weg zur europäischen Souveränität genannt: die Weiterentwicklung des Euros zu einer Währung des Welthandels. Um dorthin zu gelangen, muss die EU noch einen weiten Weg gehen. Doch selbstbewusste Europäer dürfen diesen Anspruch nicht aufgeben, sie müssen die globale Welt mitgestalten - auch mit der eigenen Währung.

\section{Und die klassische Sicherheitspolitik?}

Diese bisherigen Ausführungen über Klimaschutz, Demokratie, Handel, Digitalisierung oder Industriepolitik zeigen, wie breit und umfassend der Grüne Sicherheitsbegriff ist. Doch auch die klassische Sicherheitspolitik braucht eine intensive Befassung. Und an dieser Stelle stellt sich die übliche Frage nach der Positionierung zum Militär an und für sich und zu einzelnen Einsätzen der Bundeswehr im Besonderen. Wir setzen auf das Primat der Politik und auf zivile Krisenbearbeitung. Diese brauchen einen besseren institutionellen Unterbau, mehr Analysekapazitäten, Regionalkompetenz, Wirkungsforschung, einen intensivierten Wissenstransfers zwischen Wissenschaft, Praxis und Politik und der unmittelbaren Verfügbarkeit von Personal und Material. Auch auf europäischer Ebene. Das allgemeine Gewaltverbot der UNCharta war eine große Errungenschaft für die Menschheit. UN-geführte Friedenseinsätze sind ein zentrales Instrument kollektiver Friedenssicherung und als solche trotz aller Defizite - gerade durch eine größere europäische Beteiligung an Blauhelmeinsätzen - zu stärken. Wen der letzte Satz überrascht, weil er nicht bereit ist, vierzig Jahre alte Klischees über die Grünen abzulegen, dem sei mit einem Absatz aus dem frisch beschlossenen Grundsatzprogramm der Partei geholfen:

Die Bundeswehr ist eine im Grundgesetz und in internationalen Bündnissen verankerte Parlamentsarmee. Daraus erwächst eine Fürsorgepflicht des Parlaments gegenüber den aktiven und ehemaligen Soldat*innen und Zivilbeschäftigten sowie die Verpflichtung, sie entsprechend ihrem Auftrag und ihren Aufgaben personell und materiell auszustatten. Der Auftrag und die Aufgaben der Bundeswehr orientieren sich an den realen und strategisch bedeutsamen Herausforderungen für Sicherheit und Friedenssicherung. Sie ist ein notwendiges Mittel staatlicher und internationaler Sicherheitspolitik.

Eine wesentliche Säule der Grünen Außen- und Sicherheitspolitik ist der Feminismus. Die gleichberechtigte Mitwirkung von Frauen in den internationalen Beziehungen, Mitbestimmung an diplomatischen Verhandlungen auf Augenhöhe oder bei der Zusammensetzung internationaler Institutionen ist dabei von großer Bedeutung. Auch die feministische Außenpolitik folgt dabei dem Leitmotiv Grüner Politik: der menschlichen Sicherheit.

Dazu gehört auch eine restriktivere Rüstungsexportpolitik und die konsequente Umsetzung der Rüstungsexportrichtlinien, die Exporte von Waffen und Rüstungsgütern an Staaten, die die Menschenrechte systematisch verletzen, untersagt. Auch hier braucht es eine gemeinsame restriktive Rüstungsexportkontrolle der EU mit starken Institutionen und in EU-Gemeinschaftsrecht gegossene Exportkriterien. Rüstungs- 
kontrolle und Abrüstung sind nicht nur Instrumente der Friedenspolitik, sondern auch der Sicherheitspolitik.

Zur Abrüstung gehört auch, die Chance eines Neustarts der transatlantischen Beziehungen dafür zu nutzen, um mit der Biden/Harris-Regierung über Barack Obamas Vision Zero ins Gespräch zu kommen. Eine atomwaffenfreie Welt war stets und bleibt Grünes Ziel, sie ist Teil der DNA der Partei. Die Beendigung der Existenz von Waffen, die mit Leichtigkeit die Menschheit auslöschen können, bleibt zentrale Aufgabe unserer Politik. Dazu gehören auch die Beendigung der nuklearen Teilhabe Deutschlands in der NATO und ein Beitritt Deutschlands zum UN-Atomwaffenverbotsvertrag. Die NATO ist als Bündnis mittelfristig unverzichtbar, auch weil ihre Rückversicherungspolitik hilft, die EU in Zentral- und Osteuropa zusammenzuhalten. Wir Grüne wissen, dass für die Beendigung der deutschen nuklearen Teilhabe zahlreiche Gespräche im Bündnis notwendig sind, auch mit europäischen Partnerstaaten. Wir wissen auch, dass der Weg zum Abzug der Atomwaffen aus Deutschland von vielen Meilensteinen begleitet sein muss. Dazu gehören beispielsweise internationale Initiativen zur Reduzierung der Zahl von Atomwaffen, ein Verzicht der NATO auf jeden Erstschlag sowie eine breite öffentliche Debatte über die veraltete Abschreckungsdoktrin des Kalten Krieges. Dass der NEW START-Vertrag zwischen den USA und Russland verlängert wurde, ist ein bemerkenswertes und positives Zeichen in die richtige Richtung. Ziel muss es sein, auf dem Weg zu einer Atomwaffen-freien Welt nicht nur zum alten Vertragsregime des Kalten Krieges zwischen den USA und Russland zurückzukehren, sondern auch China mit einzuschließen. Es gibt dafür derzeit keine sich anbahnenden Lösungen, was aber die Politik vom Versuch nicht entpflichtet.

\section{Echte Sicherheit statt $2 \%$-Geldverbrennung}

Die Grüne Russland-Politik sieht für manche Beobachter*innen auf den ersten Blick sehr traditionell aus. Sie beachtet schlicht, dass die zu oft erfolgte öffentliche Gleichsetzung Russlands mit der Sowjetunion verfehlt ist. Auch die Grüne Haltung der NATO gegenüber ist sicher keine für eckige Schubladen. So führt für die Grünen das NATO-Ziel, das alle Mitgliedsstaaten anstreben mögen, 2\% ihres Bruttoinlandsprodukts für Verteidigungsausgaben aufzuwenden, nicht zu mehr Sicherheit, sondern zu mehr Geldverbrennung. Europäische NATO-Staaten geben derzeit dreimal so viel für Rüstung aus wie Russland. Die USA haben 30 Waffensysteme, wir in Europa 178. Das zeigt die Notwendigkeit effizienterer Strukturen im Beschaffungsbereich sowie eine dringend notwendige Vertiefung europäischer Zusammenarbeit in der Rüstung. In der Debatte um das 2\%-Ziel geht leider auch allzu oft verloren, dass wir dringender denn je mehr Ressourcen, aber vor allem mehr Personal für die deutsche Diplomatie bereitstellen müssen, statt sie für ineffiziente Rüstungsbeschaffungsmaßnahmen aufzuwenden. Es braucht eine Neujustierung der Aufgaben und der Fähigkeiten in Europa und nicht einfach nur einen immer höheren Verteidigungsetat in den europäischen NATO-Mitgliedstaaten. Mehr Geld in ineffektive Strukturen zu geben, ist mehr Verschwendung des Geldes der Steuerzahler, nicht mehr Sicherheit. Stattdessen sollten die EU-Mitgliedstaaten ihren Fokus darauflegen, ihre Ver- 
teidigungsfähigkeiten stärker zu bündeln und enger miteinander zu kooperieren. In den nächsten fünf Jahren werden wichtige Entscheidungen von globaler Bedeutung getroffen. Dabei wird es zentral auf Deutschland als kooperativer Akteur im europäischen Chor für Klimaschutz und Demokratie ankommen. Deutschland als Anwalt des Multilateralismus und Gegner nationaler Egoismen. Deutschland als Bannerträger der Menschenrechte und einer nachhaltigen globalen Entwicklung. Als starker Akteur einer globalen Solidargemeinschaft in der Bewältigung der Pandemie. Dies ist nicht nur eine Frage der Moral, sondern eine der Geostrategie, der Weltpolitikfähigkeit und der Souveränität.

Funding Open Access funding enabled and organized by Projekt DEAL.

Open Access Dieser Artikel wird unter der Creative Commons Namensnennung 4.0 International Lizenz veröffentlicht, welche die Nutzung, Vervielfältigung, Bearbeitung, Verbreitung und Wiedergabe in jeglichem Medium und Format erlaubt, sofern Sie den/die ursprünglichen Autor(en) und die Quelle ordnungsgemäß nennen, einen Link zur Creative Commons Lizenz beifügen und angeben, ob Änderungen vorgenommen wurden.

Die in diesem Artikel enthaltenen Bilder und sonstiges Drittmaterial unterliegen ebenfalls der genannten Creative Commons Lizenz, sofern sich aus der Abbildungslegende nichts anderes ergibt. Sofern das betreffende Material nicht unter der genannten Creative Commons Lizenz steht und die betreffende Handlung nicht nach gesetzlichen Vorschriften erlaubt ist, ist für die oben aufgeführten Weiterverwendungen des Materials die Einwilligung des jeweiligen Rechteinhabers einzuholen.

Weitere Details zur Lizenz entnehmen Sie bitte der Lizenzinformation auf http://creativecommons.org/ licenses/by/4.0/deed.de. 